\title{
EVALUACIÓN DE LAS PROPIEDADES QUÍMICAS Y MECÁNICAS DE BIOPOLÍMEROS A PARTIR DEL ALMIDÓN MODIFICADO DE LA PAPA
}

\author{
Alarcón Cavero, Hugo Arturo ${ }^{1,2}{ }^{*}$ y Arroyo Benites, Edmundo ${ }^{1}$
}

\begin{abstract}
RESUMEN
En el presente artículo presentamos el estudio realizado para la obtención de biopolímeros a partir del almidón de papa, para ser utilizado como empaque de alimentos, se modificó el almidón con ácido acético al 5\% obteniéndose un polímero con un $61,76 \%$ de amilosa y $38,28 \%$ de amilopectina. Las pruebas de elongación y tracción mostraron que el almidón modificado presentó una mejor propiedad mecánica.

Asimismo, se prepararon películas poliméricas donde se adicionaron aditivos al almidón modificado para lograr que el film mejore sus propiedades mecánicas y su textura, para ello se utilizó al Chitosan y Xathan en diferentes proporciones, determinándose una mejora de la propiedad mecánica del biopolímero. Esto fue corroborado con pruebas de elongación y tracción, obteniéndose valores del $33 \%$ y $8,47 \mathrm{~N}$, respectivamente, las espectroscopias de infrarrojo y Uv-Vis realizadas a los biopolímeros muestran picos característicos de celulosa y una buena transmitancia del $87 \%$. Todas las muestras fueron obtenidas siguiendo el método Taguchi.
\end{abstract}

Palabras clave: Biopolímeros, biodegradables, almidón de papa, empaques de alimentos.

\section{Evaluation of chemical and mechanical properties of biopolymers by modified potato starch}

\begin{abstract}
In this paper we present the study to obtain biopolymers from potato starch, for use as a food packaging, starch with acetic acid was changed to $5 \%$ yielding a polymer with a $61.76 \%$ amylose and 38.28\% amylopectin. The elongation and tensile tests showed that the modified starch presented better mechanical property.

Also, additives were added to the films by modified starch for to make the film improved mechanical properties and texture for this to Xathan Chitosan and were used in different

\footnotetext{
${ }^{1}$ Docente investigador Universidad de Lima. Av. Javier Prado Este 4600. Monterrico - Surco. Lima. halarcon@ ulima.edu.pe

${ }^{2}$ Docente investigador Facultad de Ciencias - Universidad Nacional de Ingeniería
} 
ratios, determining an improved mechanical property of the biopolymer were prepared. This was corroborated by elongation and tensile tests, yielding values of $33 \%$ and 8.47 $\mathrm{N}$ respectively and the Infrared spectroscopy -vis made Uv showing characteristic peaks biopolymers cellulose and good transmittance of $87 \%$. All samples were obtained following the Taguchi method.

Key words: Biopolymers, biodegrade, potato starch, food packaging.

\section{INTRODUCCIÓN}

Actualmente, los envases o empaques han permitido mejorar la calidad de vida del hombre, han contribuido en la comercialización de todo tipo de productos, garantizando la calidad de los alimentos cuando se requiere almacenarlos y han generado un nivel de desarrollo económico en todos los países; sin embargo, lo negativo de este desarrollo es en el impacto ambiental ${ }^{1}$. Los envases conforman un componente imprescindible de los sistemas de distribución de los alimentos y contribuyen en poner una gran variedad de productos al alcance del consumidor. La existencia del producto y empacado co-ayuda de manera importante a reducir los tiempos requeridos para la compra y preparación de los alimentos, traduciéndose en la búsqueda continua de esquemas de disminución de envolturas y envases ${ }^{2}$. Podemos definir envase como: "Todo producto fabricado con cualquier material que se utilice para contener, proteger, manipular, distribuir y presentar mercancías, desde materias primas hasta artículos acabados y desde el fabricante hasta el usuario o consumidor final. Los objetos desechables con estos fines se consideran también envases"3.

La remoción y disposición final de materiales orgánicos sintéticos, a partir del petróleo, ha generado problemas medioambientales de gran significancia. Por ello, actualmente, el almidón es uno de los componentes más importante para la fabricación de estos materiales de empaque, que contribuye a un mejor control ambiental ${ }^{4}$.

En el análisis de sostenibilidad, en el Perú, la papa es la mejor alternativa para obtener el almidón debido a que su producción duplica el consumo orientado a la canasta familiar (aproximadamente 2000 en miles de TM) y que aumenta sosteniblemente su producción, a diferencia del maíz y la yuca donde su producción tiene un aumento poco significativo ${ }^{5}$. El almidón de la papa está constituido por glucosa dispuestas en dos componentes: amilosa y amilopectina; su proporción varía de un tipo a otro según sea su fuente. El contenido de amilosa y el grado de polimerización son importantes en la determinación de las propiedades físicas, químicas y funcionales del almidón. Por ejemplo, el tamaño de los gránulos del almidón muestra una relación con la proporción amilosa/amilopectina ${ }^{6}$. Para mejorar las propiedades mecánicas del almidón es común someterlo a modificaciones, tanto físicas como químicas. El almidón que contiene amilopectina, alcanza valores más bajos de torque al ser procesado mediante extrusión ${ }^{7}$. Un alto contenido en amilosa provoca mayor fuerza de atracción entre los granos de almidón, esto impide la penetración de agua dentro de los gránulos, retrasando lógicamente el proceso de gelatinización durante el proceso de extrusión. El uso del almidón 
sin modificar está muy limitado debido a sus pobres propiedades, como son: el deterioro de las propiedades mecánicas por la exposición a la humedad, la reducida procesabilidad debido a su alta viscosidad y la fragilidad de las películas fabricadas. Por estas limitaciones citadas, se realiza una modificación química del almidón que consiste en reemplazar el grupo hidroxilo de las moléculas del almidón, por algún grupo éster o éter ${ }^{8}$. Entre las mejoras que se debe considerar en la obtención de biopolímeros como empaque de alimentos, es la de utilizar aditivos específicos en las cantidades apropiadas, con el objetivo de mejorar sus propiedades. Se puede mejorar la maquinación agregando polivinilalcohol o glicerina, elaborando películas por procesos de extrusión, compresión y otras operaciones térmicas. El quitosano ha sido muy utilizado en la composición de films y revestimientos antimicrobianos por su acción bactericida y fungicida, debido a su capacidad de ligarse a las moléculas del agua e inactivar las enzimas microbianas. Si al biopolímero obtenido del almidón, se le agrega quitosano, aumentaría su propiedad mecánica y de barrera, así como evitaría la generación de hongos y bacterias en la superficie del material, debido a las propiedades antifúngicas y antimicrobianas, prolongando de esta forma el tiempo de vida de los alimentos. El porcentaje de estos componentes varían y están en relación con el material alimenticio en estudio ${ }^{9}$. Asimismo, la goma Xanthan, es un polisacárido natural de alto peso molecular, gracias a la rigidez estructural de la molécula, produce varias propiedades funcionales inusuales como estabilidad al calor, tolerancia de soluciones fuertemente agrias y básicas, viscosidad estable en un rango amplio de temperatura, y resistencia a la degradación enzimática ${ }^{10}$.

El presente trabajo tiene como objetivo obtener un biopolímero que pueda ser utilizado para empaque de alimentos, que cumpla las normas requeridas para dicho propósito, por lo que mejorar las propiedades mecánicas y de barrera constituye el propósito fundamental, y para obtener estos beneficios se requiere tener un almidón modificado químicamente, donde la proporción de amilosa aumente, así también añadir aditivos que mejoren dichas propiedades y mantener la inocuidad de los alimentos. Por otro lado, la modificación física del almidón permite obtener un entrecruzamiento molecular y constituir películas delgadas con mayor resistencia a la tensión y elongación, esto se desarrolla aplicando temperaturas apropiadas en las diferentes etapas del proceso de fabricación del biopolímero; para ello se buscó temperaturas y tiempos óptimos para la obtención de los films. 


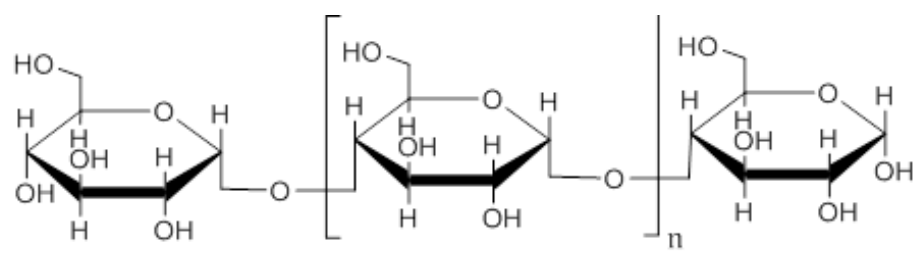

amilosa

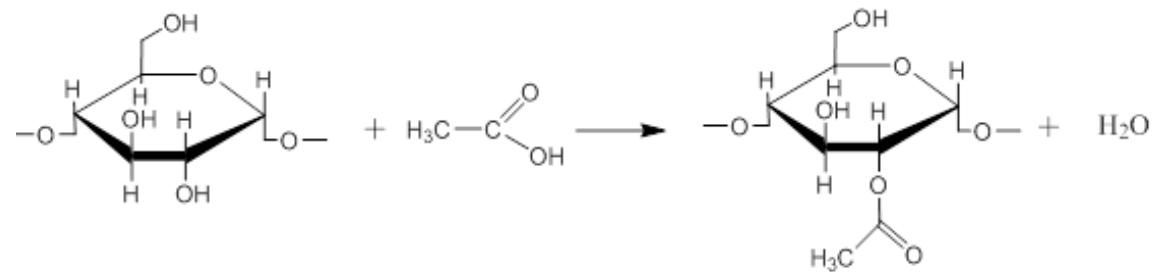
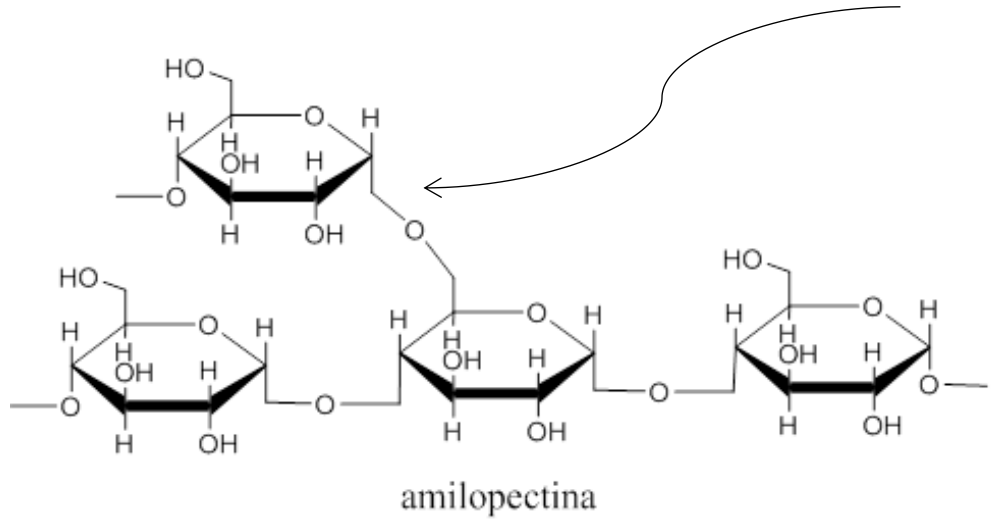

\section{PARTE EXPERIMENTAL}

\section{Preparación de láminas de biopolímeros}

\subsection{Materiales y equipos}

Para la obtención de los biopolímeros se utilizaron las siguientes sustancias: almidón de papa, agua destilada, alcohol polivinílico (PVA), etanol, glicerina, hidroxiletilcelulosa (Hxcel), cloruro de sodio $(\mathrm{NaCl})$, ácido acético y ácido bórico, Chitosan y goma Xanthan.

\subsection{Métodos de obtención}

La obtención de un biopolímero que sirva como empaque de alimentos a partir de almidón de papa, requiere una preparación que permita controlar las condiciones en cada etapa de su proceso, así como las cantidades apropiadas de los reactivos que aportan características para este nuevo material. Para controlar las condiciones en cada etapa del proceso se diseñó un biorreactor, que de manera automática mantenga las condiciones del proceso de manera 
óptima. La experiencia se realizó utilizando almidón sin modificar -almidón natural- sin tratamiento con ácido acético, y almidón modificado, se utilizó la muestra tratada con ácido acético porque es la que alcanzó la mayor cantidad de amilosa. Los reactivos utilizados que permitieron controlar la inocuidad, así como mejorar las propiedades mecánicas, fueron el Chitosan y el goma Xanthan.

\subsubsection{Preparaciones preliminares y estandarización del procedimiento}

Para determinar el número de pruebas experimentales se aplicó el método Taguchi ${ }^{11}$, el mismo nos permite obtener la combinación más adecuada y robusta de los componentes a utilizar.

Las cantidades obtenidas serán el estándar en los procedimientos para obtener el biopolímero, utilizando almidón natural (sin modificar) y almidón modificado de la papa.

Se determinó el siguiente modelo: $\mathbf{L}_{9}(\mathbf{3})^{\mathbf{2}}$

Dónde:

$\mathbf{a}=\mathbf{9}$, Se desarrollan 9 pruebas experimentales

$\mathbf{b}=\mathbf{3}$, Son los niveles para cada factor a analizar: Alcohol polivinílico (PVA), Hidroxilcelilosa (Hcel) y Glicerina (Glicer), para cada caso: alto, moderado y bajo $c=2$, Son efectos independientes: Tipo de plastificante, cantidad (\%masa, gramos, $\mathrm{mL}$ )

\subsubsection{Obtención del biopolímero con almidón natural:}

La obtención del biopolímero se realizó de acuerdo con el siguiente diagrama:
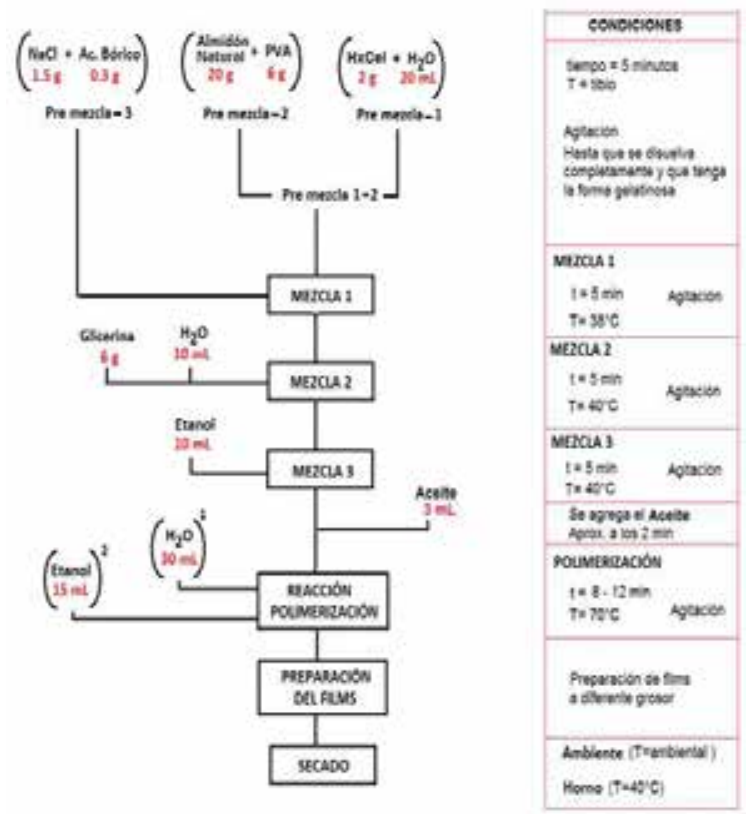

Diagrama 1. Procedimiento para la obtención de biopolímero a partir del almidón natural (no modificado) 
$\left(0^{1}\right.$ : Cuando la mezcla llega a $\operatorname{los} 70^{\circ} \mathrm{C}$ se añade de 10 en $10 \mathrm{~mL}$ el agua, según como se va gelatinizando y evitar que se endurezca.

$0^{2}$ : Cuando la mezcla llega a los $70^{\circ} \mathrm{C}$ se añade de 5 en $5 \mathrm{~mL}$ de etanol, con el objetivo de terminar de disolver los grumos y la mezcla se vuelve transparente.

Tabla 1. Cantidades estandarizadas de los diferentes componentes

\begin{tabular}{|c|c|c|c|c|c|c|}
\hline $\begin{array}{c}\text { PolivVinil } \\
\text { Alcohol } \\
(\mathbf{g})\end{array}$ & $\begin{array}{c}\text { Hidroxil } \\
\text { celelulosa } \\
(\mathbf{g})\end{array}$ & $\begin{array}{c}\text { Glicerina } \\
(\mathbf{m L})\end{array}$ & $\begin{array}{c}\text { Agua } \\
(\mathbf{m L})\end{array}$ & $\begin{array}{c}\text { Aceite } \\
\text { Resina } \\
(\mathbf{m l})\end{array}$ & $\mathbf{N a C l}(\mathbf{g})$ & Ácido Bórico (g) \\
\hline 6 & 2 & 6 & 60 & 3 & 1,5 & 0,3 \\
\hline
\end{tabular}

Tabla 2. Descripción de las muestras de biopolímero

\begin{tabular}{|l|l|l|}
\hline Muestras & \multicolumn{1}{|c|}{ Almidón modificado } & Componentes \\
\hline M1 & $20 \mathrm{~g}$ tratado con el $15 \%$ Ac. Acético & Estándares \\
\hline M2 & $20 \mathrm{~g}$ tratado con el $10 \%$ Ac. Acético & Estándares \\
\hline M3 & $20 \mathrm{~g}$ tratado con el $5 \%$ Ac. Acético & Estándares \\
\hline
\end{tabular}

\subsubsection{Obtención del biopolímero con almidón modificado:}

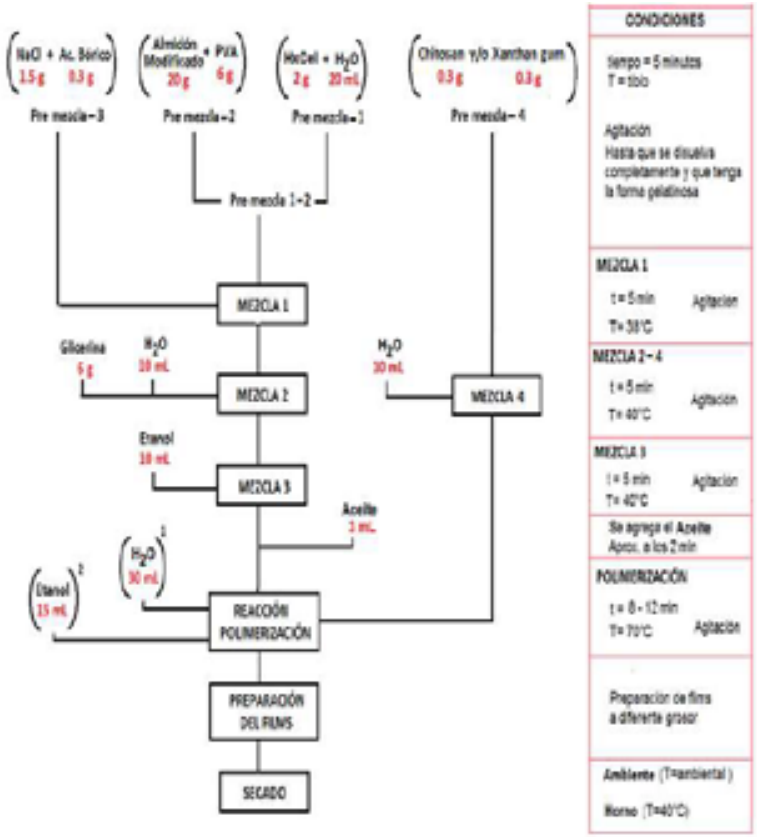

Diagrama 2. Procedimiento para la obtención de biopolímero a partir del almidón modificado 
Tabla 3. Descripción de las sustancias para las muestras de biopolímero con aditivos

\begin{tabular}{|c|c|c|c|c|}
\hline Muestras & $\begin{array}{c}\text { Almidón } \\
\text { modificado }\end{array}$ & Componentes & Chitosan & $\begin{array}{c}\text { Xanthan } \\
\text { Gum }\end{array}$ \\
\hline M4 & 20 & Cant. estándares & 0,3 & 0,3 \\
\hline M5 & 20 & Cant. estándares & 0,3 & 0 \\
\hline M6 & 20 & Cant. estándares & 0 & 0,3 \\
\hline
\end{tabular}

\subsection{Métodos de caracterización de los biopolímeros}

Medidas de elongación y tracción. Se han realizado los ensayos de caracterización mecánica de las muestras de biopolímeros a partir del almidón de papa natural y almidón de papa modificado, en el laboratorio $\mathrm{N}^{\circ} 12$ - LABICER de la Universidad Nacional de Ingeniería, Facultad de Ciencias, a condiciones ambientales (temperatura $=24^{\circ} \mathrm{C}$ y humedad relativa $=59 \%$ ). Los ensayos realizados corresponde a fuerza de tracción y elongación, para ello utilizaron la máquina de tracción universal ZWICK ROELL Z010, bajo la norma técnica ASTM D882.

Tabla 4. Pruebas de tracción y elongación sin aditivos

\begin{tabular}{|c|c|c|c|c|c|c|}
\hline Muestra & $\begin{array}{l}\text { Espesor } \\
(\mathrm{mm})\end{array}$ & $\begin{array}{l}\text { Ancho } \\
(\mathbf{m m})\end{array}$ & $\begin{array}{l}\text { Fuerza } \\
\text { máxima de } \\
\text { tracción (N) }\end{array}$ & $\begin{array}{l}\text { Elongación } \\
\text { máxima (\%) }\end{array}$ & $\begin{array}{l}\text { Relación } \\
\text { Elongación/ } \\
\text { Tracción }\end{array}$ & Norma Técnica \\
\hline M1 & 0,16 & 24,08 & 6,07 & 14,38 & 2,37 & \multirow{3}{*}{ ASTM D882 } \\
\hline M2 & 0,12 & 24,71 & 8,60 & 8,45 & 0,98 & \\
\hline M3 & 0,11 & 25,51 & 6,77 & 13,97 & 2,06 & \\
\hline
\end{tabular}

En este cuadro se observa que la muestra M3 es la más significativa, debido a que la relación elongación por unidad de tracción es 2,06 con un espesor de $0,11 \mathrm{~mm}$, mientras que la muestra M1 su relación es 2,37 pero con un espesor mucho mayor $0,16 \mathrm{~mm}$.

Tabla 5. Pruebas de tracción y elongación con aditivos

\begin{tabular}{|c|c|c|c|c|c|l|}
\hline Muestra & $\begin{array}{c}\text { Espesor } \\
\mathbf{( m m})\end{array}$ & $\begin{array}{c}\text { Ancho } \\
\mathbf{( m m}\end{array}$ & $\begin{array}{c}\text { Fuerza } \\
\mathbf{m a ́ x i m a} \text { de } \\
\text { tracción (N) }\end{array}$ & $\begin{array}{c}\text { Elongación } \\
\text { máxima (\%) }\end{array}$ & $\begin{array}{c}\text { Relación } \\
\text { Elongación / } \\
\text { Tracción }\end{array}$ & Norma Técnica \\
\cline { 1 - 5 } M4 & 0,13 & 24,87 & 8,47 & 33,00 & 3,90 & \multirow{2}{*}{ ASTM D882 } \\
\cline { 1 - 5 } M5 & 0,13 & 25,19 & 11,60 & 25,10 & 2,16 & \\
\hline M6 & 0,11 & 25,35 & 10,80 & 24,90 & 2,31 & \\
\hline
\end{tabular}

En este cuadro se observa que la muestra M4 es la más significativa, ya que la relación elongación por unidad de fuerza es de 3,90, el cual es mucho mayor que las muestras M5 y M6 donde su relación es 2,16 y 2,31, ya que el aumento es en 80,6\% y 54,8\% por unidad de fuerza, respectivamente. Respecto a los espesores estos están casi en la misma relación. 


\subsubsection{Ensayos por espectroscopia infrarroja}

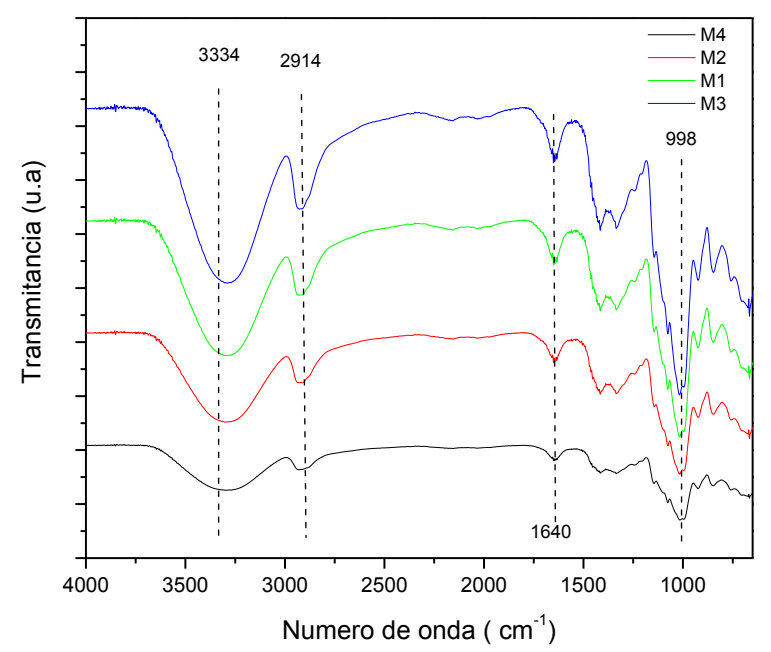

En todos los casos, los espectros establecen como biopolímero a la celulosa de acuerdo a las bandas características de los grupos $\mathrm{OH}$ en la región de $3000 \mathrm{~cm}-1$; asimismo, la banda de las vibraciones C-H a $2914 \mathrm{~cm}^{-1}$ y las vibraciones a 1640 y $998 \mathrm{~cm}^{-1}$ las cuales están relacionadas a la hemicelulosa y a las pectinas.

Las pruebas de transmitancia (no se presenta el gráfico) muestran un valor en promedio del $87 \%$, lo que indica una buena transmitancia respecto al paso de la luz.

\section{CONCLUSIONES}

La obtención de un almidón modificado con buenas propiedades mecánicas, de acuerdo a pruebas realizadas, se logra por la adición de ácido acético al $5 \% \mathrm{v} / \mathrm{v}$ debido a que mejora la relación de amilopectina y amilosa, la adición de quitosano y goma Xantan le brindan una mejor textura al biopolímero, además de mejorar la propiedad mecánica en un 4\%, esto fue corroborado con las pruebas de elongación y tracción.

Las caracterizaciones por espectroscopia infrarroja muestran que la adición de aditivos no cambia las características estructurales del biopolímero; asimismo, la prueba de transmitancia muestra un valor bastante aceptable de transparencia del biopolímero.

\section{AGRADECIMIENTOS}

A la Facultad de Ingeniería Industrial y al Instituto de Investigación Científica de la Universidad de Lima (IDIC) por el financiamiento otorgado para la realización del presente trabajo. 


\section{REFERENCIAS BIBLIOGRÁFICAS}

1. Avendaño GC. Diseño y evaluación de las propiedades mecánicas y de barrera de un biopolímero obtenido a partir de almidón de papa para ser empleado en empaques para alimentos. Duitama: Universidad Nacional Abierta y a Distancia UNAD, Escuela de Ciencias Básicas e Ingeniería, Ingeniería de Alimentos; 2009.

2. Tubón IR. Formulación, elaboración y evaluación de bioenvase para caramelos a base de almidón de yuca, sacarosa y gelatina. Riobamba: Facultad de Ciencias de la Escuela Superior Politécnica de Chimborazo; 2013.

3. Parlamento Europeo. Directiva 94/62/CE del Parlamento Europeo y del Consejo Relativa a los envases y residuos de envases. Diario Oficial de las Comunidades Europeas; 1994: $\mathrm{N}^{\circ} \mathrm{L} 365 / 10$.

4. Enríquez M, Velasco R, Ortiz V. Composición y procesamiento de películas biodegradables basadas en almidón. Revista de Biotecnología en el Sector Agropecuario y Agroindustrial. 2012; 10(1): 182-192.

5. Arroyo EV, Alarcón HA. Obtención, caracterización y análisis comparativo de polímeros biodegradables a partir de la yuca, papa y maíz. Lima: Instituto de investigación científica de la Universidad de Lima; 2013.

6. Delpeuch F, Favier JC. Caracteristique des almidons de plantas alimentaires tropicales: action de l'alpha-amylase, gonflement et solubilité. Ann Technol Agric. 1980; 29(1):53 $-67$.

7. Meré J. Estudio del procesamiento de un polímero termoplástico basado en almidón de patata amigable con el medio ambiente. Madrid: Universidad Carlos III de Madrid; 2009.

8. Peñaranda OI, Perilla JE, Algecira NA. Revisión de la modificación química del almidón con ácidos orgánicos. Revista de ingeniería e investigación de la Universidad Nacional de Colombia. 2008; 28(3): 47-52.

9. Durango AM, Soares NF, Arteaga MR. Filmes y revestimientos comestibles como empaques activos biodegradables en la conservación de alimentos. Revista de Biotecnología en el sector agropecuario y agroindustrial de la Universidad de Córdova. 2011; 9(1): 112-118.

10. López CA. Xantanos biopoliméricos: propiedades reológicas y aplicaciones. Revista virtual Cienciacierta. 2011; 25.

11. Taguchi G. Introduction to quality engineering: designing quality into products and processes. Tokyo: Asian Productivity Organization; 1986. 